\title{
Utilization of the CAPS/dCAPS Method to Convert Rice SNPs into PCR-based
}

\section{Markers}

\author{
Toshiyuki Komori*1) and Naoto Nitta ${ }^{2)}$ \\ Plant Breeding and Genetics Research Laboratory, Japan Tobacco Inc., 700 Higashibara, Toyoda, Iwata, Shizuoka 438-0802, Japan \\ 1) Present address: Plant Innovation Center, Japan Tobacco Inc., 700 Higashibara, Toyoda, Iwata, Shizuoka 438-0802, Japan \\ 2) Present address: Scientific and Regulatory Affairs Division, Tobacco Headquarters, Japan Tobacco Inc., 2-2-1 Toranomon, Minato, \\ Tokyo 105-8422, Japan
}

Key Words: CAPS, dCAPS, RFLP marker, PCR-based marker, SNP, rice.

Linkage maps of rice have been constructed based on restriction fragment length polymorphism (RFLP) markers (Kurata et al. 1994, Tsunematsu et al. 1996, Harushima et al. 1998). The mapped RFLP markers have been widely used in various fields from fundamental research to practical breeding programs (Sasaki 2001, Yano 2001), and genotype data by RFLP analysis have accumulated. For example, the results of an RFLP survey among sixteen rice varieties and RFLP genotyping data of 212 doubled-haploid lines derived from the cross between Akihikari and Koshihikari have been published by the Rice Genome Research Program (RGP) (http://rgp.dna.affrc.go.jp). However, because the RFLP analysis includes time-consuming steps and requires a large amount of purified DNAs, conversion to PCR-based markers has been desired.

The cleaved amplified polymorphic sequence (CAPS) method (Konieczny and Ausubel 1993) has made it possible to develop PCR-based markers that can be rapidly and reliably analyzed. The existence of nucleotide sequence polymorphism generating a restriction site difference between varieties/lines to be analyzed is essential for developing a CAPS marker. Although single nucleotide polymorphism (SNP) is one of the most common classes of DNA polymorphism in many organisms including rice (Nasu et al. 2002, Hayashi et al. 2004), the majority of single-base changes generate no restriction site difference and thus seem useless for developing CAPS markers. However, recent research (Michaels and Amasino 1998, Neff et al. 1998) demonstrated that single-base changes generating no restriction site difference could be utilized for the development of PCRbased markers by the derived CAPS (dCAPS) method in Arabidopsis thaliana. The dCAPS method was proved to be robust for developing PCR-based markers adequate for the genetic analysis in rice whose genome is about four times

Communicated by M. Omura

Received May 31, 2004. Accepted October 26, 2004.

*Corresponding author (e-mail: Toshiyuki.Komori@ims.jti.co.jp) larger than that of A. thaliana (Komori and Nitta 2003, Komori et al. 2003, Yamanaka et al. 2004).

The recent availability of numerous rice genome sequences has promoted the survey of SNPs between varieties (Shen et al. 2004). Because SNP genotyping is useful for mapbased cloning, marker-assisted breeding, seed purity tests and so on, several new techniques including pyrosequencing (Ahmadian et al. 2000, Alderborn et al. 2000), TaqMan (Livak 1999), and fluorescence energy transfer (Chen et al. 1998) methods, for example, have been recently developed to genotype SNPs. These techniques, however, require specialized detection instruments and/or labeled oligonucleotides. In contrast, CAPS/dCAPS markers can be analyzed by simple PCR followed by restriction enzyme treatment and agarose gel electrophoresis, and therefore are still preferred in many laboratories including breeding stations.

RGP converted 171 RFLP markers that detected sequence polymorphisms between Nipponbare (japonica rice variety) and Kasalath (indica rice variety) into 165 CAPS markers and six dCAPS markers and released the information on the developed markers (http://rgp.dna.affrc.go.jp). In a preliminary study, some of the 171 PCR-based markers were used to analyze Asominori (japonica rice variety) and IR24 (indica rice variety), and nearly half of the tested markers did not show any clear polymorphisms with respect to the gel electrophoresis profiles, which strongly suggested that further development of PCR-based markers is necessary to more efficiently perform a genetic analysis for a wide range of variety/line combinations.

In this study, the genome sequences corresponding to 53 RFLP probes were compared between Asominori and IR24, and 33 PCR-based markers consisting of nine CAPS markers, 21 dCAPS markers and three amplicon length polymorphism (ALP) markers have been developed. The successful conversion from RFLP markers into PCR-based markers demonstrated the propriety of the strategy. The effect of mismatch positions in designing primers by the dCAPS method is also described. 
Development of 33 PCR-based markers based on RFLP markers

Total DNA was extracted from the green leaves of Asominori and IR24 by the CTAB method (Murray and Thompson 1980). Using the total DNA as templates, PCR was performed with sequence tagged site (STS) primers that were designed on the basis of the end sequences of 53 RFLP probes. As regards the 53 RFLP markers, STS information was obtained mainly from the RGP website (http:// rgp.dna.affrc.go.jp), but CAPS/dCAPS information was unavailable or inadequate for clear discrimination between Asominori and IR24. These RFLP markers were selected without performing RFLP analysis between Asominori and IR24. After electrophoresis of the PCR products on agarose gel, the DNA fragments of interest were excised and purified with SUPREC ${ }^{\mathrm{TM}}-01$ (TaKaRa). End nucleotide sequences were determined with the STS primers using dRhodamine Terminator Cycle Sequencing FS Ready Reaction Kit (Applied Biosystems) and an automatic DNA sequencer (Prism 310, Applied Biosystems). Sequence analyses were conducted with Genetyx-Mac version 10.0 (Software Development).
As a result of the sequence comparisons between Asominori and IR24, some degree of sequence polymorphism was observed in 33 RFLP probe regions out of 53. Insertions/deletions (InDels) consisting of more than 17 nucleotides were identified in three regions (S11114, C797 and R887), and ALP markers were developed utilizing the InDels. Although only SNP(s) and/or small InDels consisting of less than nine nucleotides were identified in 30 RFLP probe regions, nine CAPS markers and 21 dCAPS markers were developed utilizing SNPs in these regions. The dCAPS method was employed mainly when it was difficult to employ the CAPS method because of the lack of adequate SNP and/or bothersome restriction site(s) adjacent to a potential target SNP. In addition, the dCAPS method was strategically used in some cases in order to develop reliable PCR-based markers, as described below by the example of C239 MboI. The PCR-based markers developed in this study are listed in Table 1 .

Marker analysis was conducted in the following way. PCR was carried out in $30 \mu \mathrm{l}$ reactions that consisted of $15 \mathrm{ng}$ total DNA, $0.2 \mathrm{mM}$ of each dNTP, $0.2 \mu \mathrm{M}$ of each primer, 1 $\times$ Ex Taq Buffer with $\mathrm{Mg}^{2+}$ (TaKaRa) and 1.5 U TaKaRa Ex $\mathrm{Taq}^{\mathrm{TM}}$ (TaKaRa). After preheating at $94^{\circ} \mathrm{C}$ for $2 \mathrm{~min}, 35$

Table 1. List of PCR-based markers developed in this study

\begin{tabular}{|c|c|c|c|c|c|c|c|}
\hline \multirow[t]{2}{*}{ Marker } & \multirow[t]{2}{*}{ Type } & \multirow[t]{2}{*}{ Forward primer $\left(5^{\prime}\right.$ to $\left.3^{\prime}\right)$} & \multirow[t]{2}{*}{ Reverse primer $\left(5^{\prime}\right.$ to $\left.3^{\prime}\right)$} & \multirow[t]{2}{*}{ Enzyme } & \multicolumn{2}{|c|}{$\begin{array}{c}\text { Fragment size after } \\
\text { digestion }(b p)\end{array}$} & \multirow{2}{*}{$\begin{array}{l}\text { Chromo- } \\
- \text { some }\end{array}$} \\
\hline & & & & & Asominori & IR24 & \\
\hline C970 BclI & dCAPS & CAATTATAAGTGCTTCTGGAGCAATCC & GAGGGGATAGGTTGAACAGAAAtGATC & $B c l \mathrm{I}$ & 154 & 127,27 & 1 \\
\hline C955 Tsp509I & CAPS & TGGATCCTGCAAACAAAGGCGCTC & TCTACACAGAACAAGAGGCATCTC & Tsp509I & 161,158 & 318 & 1 \\
\hline S2523 HhaI & dCAPS & CTCTACATGAAGAGGTATCCAGCC & TGATGACAGCGAAGAAGAGCGcGGGTG & HhaI & 231,19 & $177,54,19$ & 1 \\
\hline S10581 AluI & CAPS & GAGAGATTGCCTGGAGGAGTAACC & GAGAGCGCCTTCACATCGTAAAGG & $A l u \mathrm{I}$ & $317,193,72,31$ & $510,72,31$ & 1 \\
\hline S10012 MspI & dCAPS & AGTGACCTCCCTCTGGGTCCTCAGA & GAAATGTCAACAAACACTGTTCAATTcCG & Msp I & 146 & 118,29 & $1^{1)}$ \\
\hline R1843 BslI & dCAPS & TAGGTAAGAAATCAAATCCAcCAACCG & GACTTGCCCATTATTTGATACcGCTAG & $B s l \mathrm{I}$ & 110,24 & $83,27,24$ & 2 \\
\hline C1496 DraI & dCAPS & TGAGGCATGTCTTGTTATCTTTGtTTA & CCACAGTTGTAGTTTGTCTCCAGG & DraI & 121,26 & 147 & $2^{2)}$ \\
\hline R3361 NdeI & CAPS & GTACGGTAATGGAGGTGGAAATGC & CAATATCTTTGCTCATAGGACTCC & $N d e \mathrm{I}$ & 276 & 216,60 & 3 \\
\hline C582 AluI & dCAPS & TTTTGTGCCTCCACCACAGCAAGG & TCCTTTTGGCAAGGAACATTCAgCTGC & $A l u \mathrm{I}$ & $98,47,23$ & 145,23 & 3 \\
\hline R273 HhaI & CAPS & AGGCTGCCTTAGGTGGTCTTCTCG & TCGCTCCTCACGATGTGCGGAGAG & $H h a \mathrm{I}$ & 129,32 & $99,32,30$ & 3 \\
\hline $\mathrm{R} 374 B s t \mathrm{UI}$ & dCAPS & AATCTGTGATTTACTGCTGATGAGCcG & TCCTGCGAGCGGCATTTGAGAACC & $B s t \mathrm{UI}$ & $45,27,25$ & 72,25 & 4 \\
\hline R3166 BslI & dCAPS & GCTGTTGCCCAAGAAAGAACAcCACCG & TTTTTGGCAGCAGCATCTccAGAGGAC & $B s l \mathrm{I}$ & 106,28 & $84,28,22$ & 5 \\
\hline $\mathrm{R} 1838$ DdeI & dCAPS & ATTTATCATCAAATGAATGCTTCcTGA & GAACATGCATAACAGACGGGGcTGAGC & $D d e \mathrm{I}$ & $92,25,24$ & 116,25 & 5 \\
\hline C235 BstPI & dCAPS & ATGTTTGCACTGCTATAAGTTgGTGAC & TTGCGAGATCCTAACACAATGAGG & Bst $\mathrm{PI}$ & 83,22 & 105 & 6 \\
\hline R2123 RsaI & dCAPS & CATGGGAACAATGTTGATTGATTGC & GCAGCACAAGTTATCAGCTTGGCAAgT & $R s a \mathrm{I}$ & 137 & 110,27 & 6 \\
\hline C81 EcoRI & dCAPS & GGTACCAGTAACTGCATGATGCgAATT & CGGATGACAGATTAACACGAGCTC & EcoRI & 106 & 83,23 & 6 \\
\hline S10324A MslI & dCAPS & ATGGGTTTATAGTGTACGGAGATC & ATTATAAACAAGATAGAAACaCCTGAG & MslI & 117 & 93,24 & 6 \\
\hline R2829 Tsp509I & dCAPS & CCGATGAAGACGGTTCATGGCATGAaA & ACAGATTGACTACCGCTTGCAaTTGGC & Tsp509I & $139,25,24$ & 164,24 & 7 \\
\hline C1467 BsrGI & dCAPS & GTGATTTTGCTTGGTGTAATAAtGTAC & GGGAATAAACAAAGGATAGCTTCGTC & Bsr GI & 72,23 & 95 & 7 \\
\hline R2561 AluI & CAPS & AAGTGCAAAGGGTGTTCCACCAGC & GATGAAGAAATGACCTCCTTGTGG & $A l u \mathrm{I}$ & $146,69,23$ & 215,23 & 7 \\
\hline C847 Tsp509I & dCAPS & GCGATCATGTTTCATAATGAcATTGACTC & CACAAACCACCCTTCCATACAGCA & Tsp509I & 156,31 & $118,38,31$ & 7 \\
\hline C924 Tsp509I & CAPS & GAAGCTCAGTTGATTGATGTTCGG & CCAAGTTCCAAACTGTCGAAGAGG & Tsp509I & 408,79 & $254,154,79$ & 7 \\
\hline R1394A HinfI & dCAPS & TGGATTGCATTCTGTTTTGGCAGC & TTTCTGCGTCCCTGATATGTGATAgAT & HinfI & 158 & 130,28 & 8 \\
\hline S11114 ALP & ALP & TGCTGGTGAGAAAATTACTGGCGG & ATGATAGCACAAAAACACACAAGC & - & 159 & 141 & 8 \\
\hline R2638 Tsp509I & CAPS & AAGGTATGCGTTCCCTTGGTTGCT & TCGCCAGGATTCTTGATCCCGCTG & Tsp509I & 90,35 & 125 & 9 \\
\hline C913A XbaI & dCAPS & TTCAACAGCTTCTTAGGATTGAGG & CCGCATGATATTCACCATCAAGTcTAG & $X b a \mathrm{I}$ & 86,27 & 113 & 10 \\
\hline C8 Tsp509I & dCAPS & GTCTCTGGCGAGTCATCTTCCTCC & GGGTTTGGTCCTTGTGTATATGAGaAT & Tsp509I & 106 & 77,28 & 10 \\
\hline C797 ALP & ALP & ATGAAGGTTCACAAAAGAGAGGGG & CATATTTCTTTGCCCATTGGCGAC & - & 158 & 130 & 10 \\
\hline $\mathrm{C} 239 \mathrm{MboI}$ & dCAPS & TCCAGATgGAGACCGCCAAtATCATCC & ATCATCTCATCGGACCGTGCAGaTCCG & MboI & 152,25 & $123,29,25$ & 10 \\
\hline C1116B Tsp509I & CAPS & TAAAGTGTATGATGATTACACGGG & AACCAATAGAACCGTAGAAGCTAC & Tsp509I & 126,77 & $77,67,59$ & 11 \\
\hline C950 BslI & dCAPS & GACACTGATGTTTTATCTATcCAGTCC & CAACATCCATAACAAGCAAACGAT & $B s l \mathrm{I}$ & 122 & 95,27 & 11 \\
\hline R887 ALP & ALP & GGATTTGATGAAACTTGCTACGGG & GATTCAGCCCATTACAAAAATGCC & - & 120 & 142 & 12 \\
\hline C454 Tsp509I & CAPS & CATGGTTCTTCTCAAGTTAGTTGC & TCAGCGTAGGAAAGAGGAACTTGC & Tsp509I & 134,28 & $110,28,24$ & 12 \\
\hline
\end{tabular}

1) Original RFLP marker S10012 is located on Chromosome 7 (Harushima et al. 1998).

2) Original RFLP marker C1496 is located on Chromosome 6 (Harushima et al. 1998). 
PCR cycles $\left(94^{\circ} \mathrm{C}\right.$ for $0.5 \mathrm{~min}, 58^{\circ} \mathrm{C}$ for $0.5 \mathrm{~min}$ and $72^{\circ} \mathrm{C}$ for $0.5 \mathrm{~min}$ ) were performed. The last cycle was followed by a final 2-min incubation at $72^{\circ} \mathrm{C}$. For CAPS and dCAPS markers, aliquots $(10 \mu \mathrm{l})$ of the PCR products were digested for $3 \mathrm{~h}$ in $20 \mu \mathrm{l}$ total volume with 5-15 units of the appropriate restriction enzyme. Then, the reactions were separated by electrophoresis on 3\% MetaPhor ${ }^{\mathrm{TM}}$ Agarose (FMC BioProducts) gel in Tris-acetate EDTA (TAE) buffer and detected by ethidium bromide staining. The pBR322 DNA-MspI digest (New England BioLabs) was used as a DNA size marker.

All 33 markers showed clear polymorphism between Asominori and IR24 with respect to the gel electrophoresis profiles, and were subjected to mapping using 71 recombinant inbred lines (RILs) derived from the cross between

A

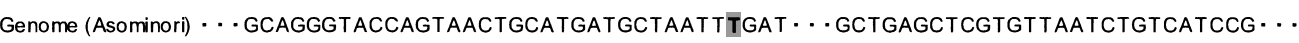
Genome (IR24) …GCAGGGTACCAGTAACTGCATGATGCTAATTCGAT * . GCTGAGCTCGTGTTAATCTGTCATCCG . .

$\underset{\text { F1 GCAGGGTACCAGTAACTGCATGATGCg }}{\stackrel{\text { Forward primer }}{\text { F2 CAGGGTACCAGTAACTGCATGATGCgA }}}$
F3 AGGGTACCAGTAACTGCATGATGCgAA
F4 GGGTACCAGTAACTGCATGATGCgAAT
F5 GGTACCAGTAACTGCATGATGCgAATT

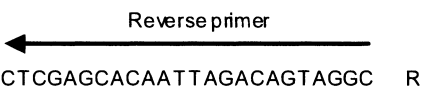

F1×R GCAGGGTACCAGTAACTGCATGATGCAATTTGAT * -GCTGAGCTCGTGTTAATCTGTCATCCG

F2×R CAGGGTACCAGTAACTGCATGATGCgAATTTGAT ‥GCTGAGCTCGTGTTAATCTGTCATCCG

F3×R AGGGTACCAGTAACTGCATGATGCgAATTTGAT . .GCTGAGCTCGTGTTAATCTGTCATCCG

F4×R GGGTACCAGTAACTGCATGATGCgAATTTGAT - . GCTGAGCTCGTGTTAATCTGTCATCCG

F5 $\times R$ GGTACCAGTAACTGCATGATGCgAATTTGAT - . GCTGAGCTCGTGTTAATCTGTCATCCG Expected product (IR24)

F1×R GCAGGGTACCAGTAACTGCATGATGCgAATTCGAT - . GCTGAGCTCGTGTTAATCTGTCATCCG F2 $\times R$ CAGGGTACCAGTAACTGCATGATGC F3×R AGGGTACCAGTAACTGCATGATGC F4×R GGGTACCAGTAACTGCATGATGC F5 $\times$ R GTACCAGTAACTGCATGATGC
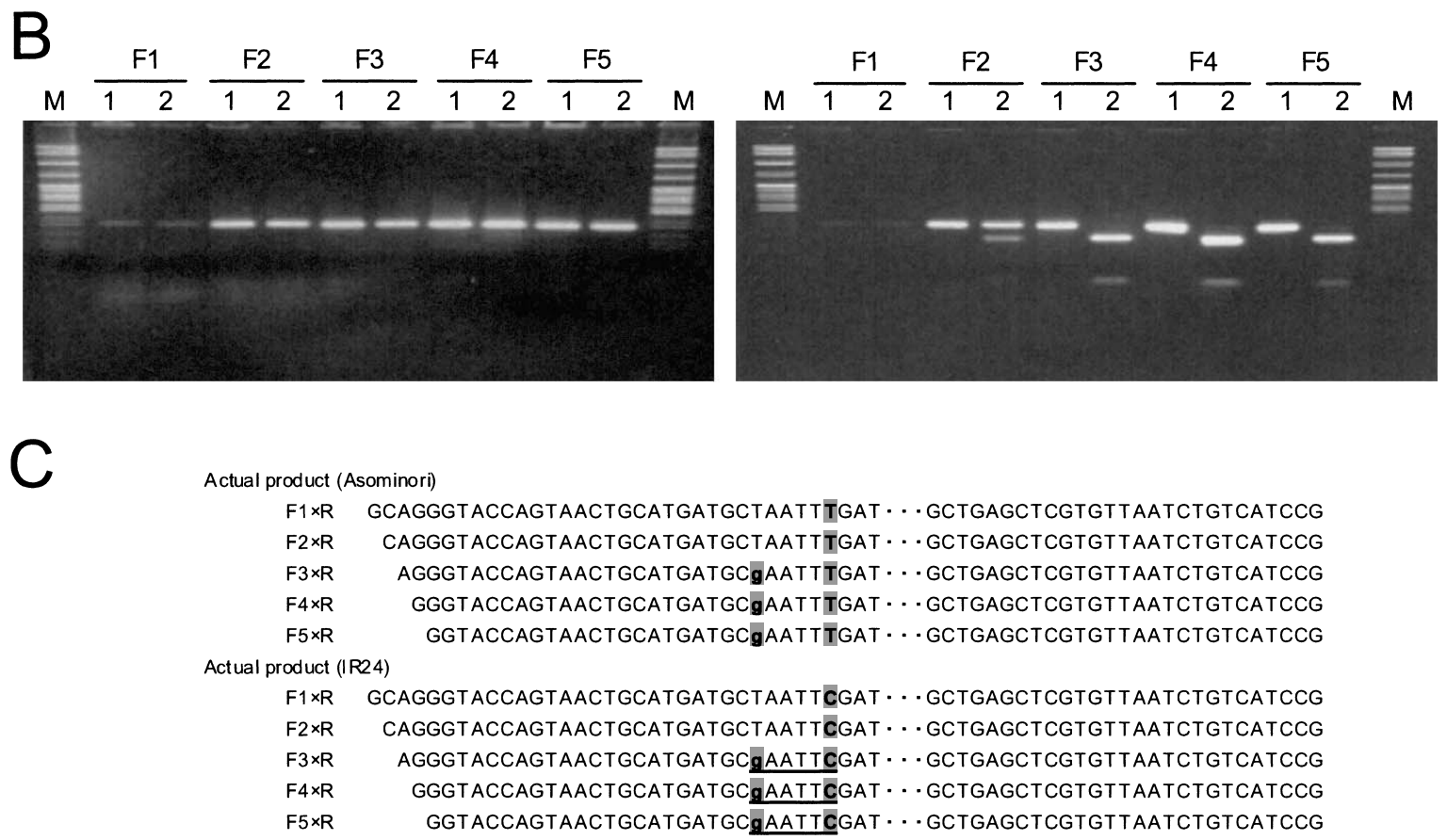

Fig. 1. Position effect of the introduced mismatch. (A) Strategy to convert an RFLP marker C81 into a PCR-based marker. The polymorphic site between Asominori and IR24 is shown in upper-case bold type. Five forward primers (F1 to F5) containing a mismatch shown in lower-case bold type were designed together with a reverse primer (R). The mismatch was expected to generate an EcoRI site (GAATTC) only in the PCR products from the IR24 DNA. (B) Agarose gel electrophoresis profiles. PCR was conducted with five primer combinations. The PCR products (left panel) and their digests with EcoRI (right panel) were separated on a 3\% MetaPhor ${ }^{\mathrm{TM}}$ Agarose gel in TAE buffer. M: DNA size marker (pBR322/ MspI digest), 1: Asominori, 2: IR24. (C) Sequence analysis of the PCR products. The sequence of each PCR product was determined by direct sequencing. 
Asominori and IR24, as previously described (Tsunematsu et al. 1996). Genotype data of the 71 RILs for 375 markers were obtained from a web site of RGP (http://rgp.dna.affrc. go.jp). Using this mapping population and the genotype data, a linkage analysis was performed with MAPMAKER version 2.0 (Lander et al. 1987) to locate the developed PCR-based markers. Among the 33 markers, 31 markers were mapped on the same chromosomal location as their corresponding RFLP marker, while two dCAPS markers (S10012 MspI and C1496 DraI) were mapped on a chromosome different from that containing the locus of their corresponding RFLP marker. The change in the mapped location probably occurred because the dCAPS markers and the RFLP markers detected different genomic regions sharing the high sequence homology. The fact that two or three bands were detected by RFLP analysis of S10012 and C1496 by RGP (http://rgp.dna.affrc.go.jp) supports this hypothesis. This change has also been observed for some CAPS markers that were developed based on RFLP markers by RGP (http://rgp.dna.affrc.go.jp). Thus, it is essential to confirm the map position of a newly developed PCR-based marker prior to its use in genetic analysis.

Of 53 RFLP probe regions where the SNP search was conducted in this study, no SNP was found in 20 regions. Because only end sequences were analyzed, SNP(s) might be found by further sequencing. If there is no SNP in the entire RFLP probe region, sequence comparison in the outer regions is necessary. Once a SNP is identified, it is highly possible to convert it into a CAPS/dCAPS marker, as demonstrated by the 30 examples in this study. It is in contrast with the allele-specific PCR method by which only 33 SNPs of 49 could be used to reliably discriminate between two alleles (Hayashi et al. 2004).

\section{Position effect of the introduced mismatch}

In the process of developing C81 EcoRI in Table 1, five forward primers (F1 to F5) that contained a mismatch in different positions were designed so that the mismatch would generate a unique $E c o$ RI site for IR24 (Fig. 1A). A reverse primer (R) was located approximately $60 \mathrm{bp}$ apart from the forward primers and contained no mismatch. Each of the forward primers was combined with the reverse primer, and PCR was carried out using the total DNA of Asominori and IR24 as templates. Figure 1B shows agarose gel electrophoresis profiles of the PCR products and their digests with EcoRI. When F3, F4 or F5 was used as a forward primer, the target fragments of approximately $110 \mathrm{bp}$ were efficiently amplified, and their EcoRI digests showed the expected pattern of electrophoresis profiles; the PCR products from IR24 DNA were cleaved with EcoRI, and those from Asominori DNA were not. In contrast, when F1 or F2 was used as a forward primer, the target fragments were less efficiently amplified, and/or, the majority of the PCR products from IR24 DNA were tolerant to EcoRI.

To clarify the reason for the unexpected results for F1 and F2, we determined the nucleotide sequences of the PCR products by direct sequencing. As shown in Figure 1C, the result demonstrated that the mismatch introduced into $\mathrm{F} 1$ and F2 was at least partially repaired during the PCR amplification, while the mismatch introduced into F3, F4 and F5 remained in the PCR products.

The effect of the position of a primer mismatch was studied further for some other cases (data not shown). In some cases, the dCAPS method worked out independent of the mismatch positions. In other cases, it worked out for the primer carrying a mismatch at the third nucleotide from the $3^{\prime}$ end, but not for the primer carrying a mismatch at the first or the second nucleotide from the $3^{\prime}$ end, presumably due to the repair of the mismatch as well as in the case of C81. Therefore, it was concluded that mismatches should be introduced at the third or more distant nucleotide from the $3^{\prime}$ end, if possible. It should be noted that the present results were inconsistent with a previous report (Michaels and Amasino 1998) that concluded that the mismatches at the first or the second nucleotide from the 3 ' end are preferable to avoid the generation of unexpected digestion-resistant PCR products. The difference might be due to the PCR conditions, especially the polymerase used. In fact, the results obtained with Y14824 MboI (Komori and Nitta 2003), one of the dCAPS markers developed by locating a mismatch at the second nucleotide from the $3^{\prime}$ end, varied with the polymerase (Fig. 2). When TaKaRa Taq ${ }^{\mathrm{TM}}$, which lacks proofreading activity, is used, the marker shows the expected pattern of electrophoresis profiles. On the contrary, when TaKaRa Ex

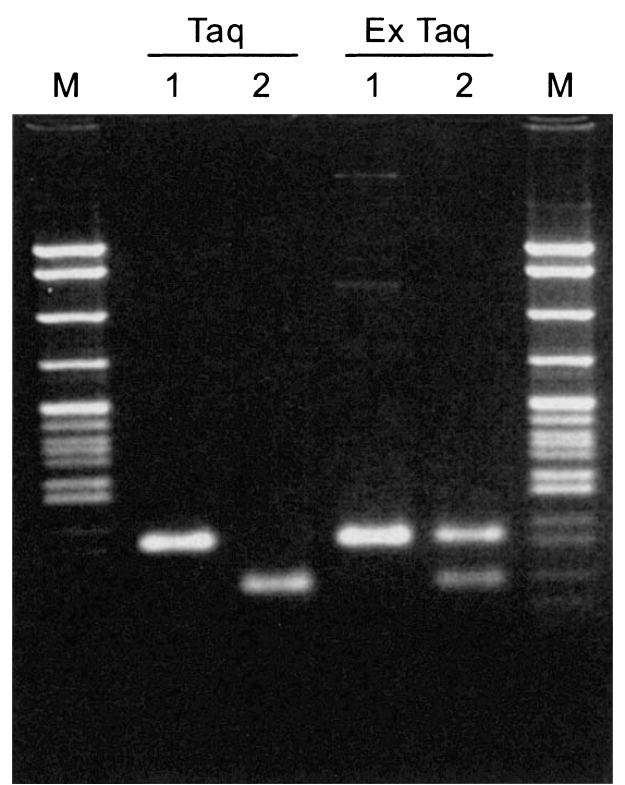

Fig. 2. The effect of proofreading activity of DNA polymerase on the agarose gel electrophoresis profiles of Y14824 MboI, a dCAPS marker developed by Komori and Nitta (2003). PCR was performed with TaKaRa $\mathrm{Taq}^{\mathrm{TM}}$ (left) or TaKaRa Ex $\mathrm{Taq}^{\mathrm{TM}}$ (right). The PCR products were digested with $\mathrm{MboI}$ and separated on a 3\% MetaPhor ${ }^{\mathrm{TM}}$ Agarose gel in TAE buffer. M: DNA size marker (pBR322/MspI digest), 1: Asominori, 2: IR24. 
A

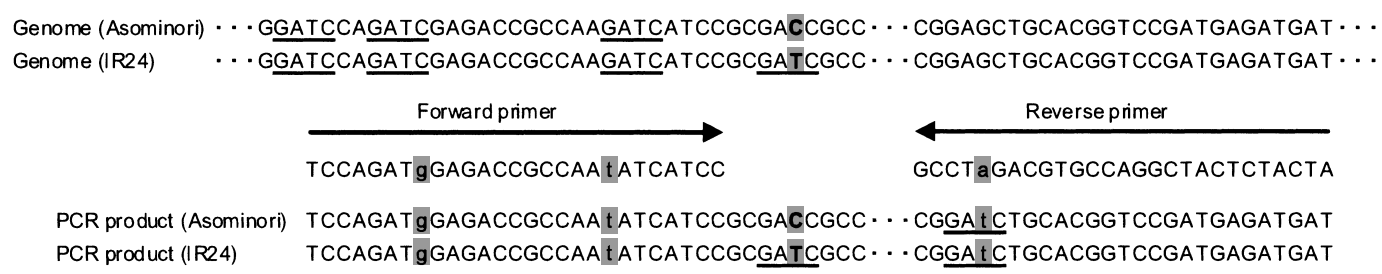

B

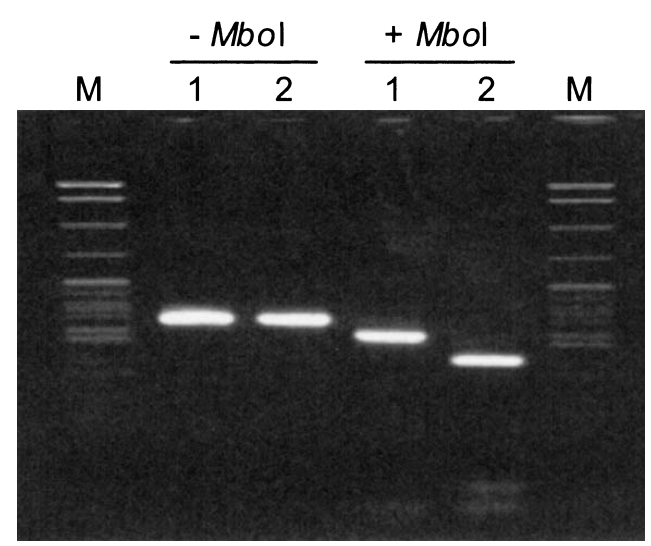

Fig. 3. Example of advanced applications of the dCAPS method. (A) Strategy to develop C239 MboI. The polymorphic site between Asominori and IR24 is shown in upper-case bold type. The forward primer contains two mismatches shown in lower-case bold type to disrupt the MboI sites (GATC). The reverse primer contains a mismatch shown in lower-case bold type to create an $\mathrm{MboI}$ site. (B) Agarose gel electrophoresis profiles. The PCR products and their digests with $\mathrm{MboI}$ were separated on a 3\% MetaPhor ${ }^{\mathrm{TM}}$ Agarose gel in TAE buffer. M: DNA size marker (pBR322/MspI digest), 1: Asominori, 2: IR24.

$\mathrm{Taq}^{\mathrm{TM}}$, which has proofreading activity and a higher amplification efficiency than TaKaRa Taq ${ }^{\mathrm{TM}}$, is used, a considerable amount of digestion-resistant PCR products is observed. It is reminded that the electrophoresis profiles of the dCAPS markers could be changeable depending on the polymerase used.

\section{Advanced application of the dCAPS method}

The dCAPS method can potentially utilize any sequence polymorphism for the development of a PCR-based marker. In addition to the primary application, the dCAPS method provides advanced applications. One typical example is shown in Figure 3A, which illustrates the strategy to develop C239 MboI in Table 1. In this case, a SNP was found in the MboI site. However, it seemed difficult to detect the SNP by the CAPS method because of the existence of $M b o I$ sites in the vicinity of the polymorphic $M b o \mathrm{I}$ site. To solve this problem, we introduced two mismatches into the forward primer so that the redundant $M b o$ I sites would be disrupted. Furthermore, a mismatch was introduced into the reverse primer to generate an $\mathrm{MboI}$ site for both alleles. The common $M b o$ I site is used to avoid mis-genotyping due to incompleteness of the restriction enzyme digestion; complete digestion can be confirmed by disappearance of the original PCR products (Fig. 3B).

\section{Acknowledgements}

We are grateful to Prof. Atsushi Yoshimura of Kyushu University for providing the seeds of 71 RILs and the Rice Genome Research Program (RGP) for providing the information on the rice RFLP clones. We also thank Ms. Yoshiko Amma and Ms. Mika Tabayashi for their technical assistance.

\section{Literature Cited}

Ahmadian, A., B.Gharizadeh, A.C.Gustafsson, F. Sterky, P.Nyren, M.Uhlen and J.Lundeberg (2000) Single-nucleotide polymorphism analysis by pyrosequencing. Anal. Biochem. 280: 103110 .

Alderborn, A., A.Kristofferson and U.Hammerling (2000) Determination of single-nucleotide polymorphisms by real-time pyrophosphate DNA sequencing. Genome Res. 10: 1249-1258.

Chen,X., K.J.Livak and P.Y.Kwok (1998) A homogeneous, ligasemediated DNA diagnostic test. Genome Res. 8: 549-556.

Harushima, Y., M.Yano, A.Shomura, M.Sato, T.Shimano, Y.Kuboki, T.Yamamoto, S.Y.Lin, B.A.Antonio, A.Parco, H.Kajiya, N.Huang, K.Yamamoto, Y.Nagamura, N.Kurata, G.S.Khush and T.Sasaki (1998) A high-density rice genetic linkage map with 2275 markers using a single $\mathrm{F}_{2}$ population. Genetics 148: 479-494.

Hayashi,K., N.Hashimoto, M.Daigen and I.Ashikawa (2004) Devel- 
opment of PCR-based SNP markers for rice blast resistance genes at the Piz locus. Theor. Appl. Genet. 108: 1212-1220.

Komori,T. and N.Nitta (2003) High frequency of sequence polymorphism in rice MITEs and application to efficient development of PCR-based markers. Breed. Sci. 53: 85-92.

Komori,T., T.Yamamoto, N.Takemori, M.Kashihara, H.Matsushima and N.Nitta (2003) Fine genetic mapping of the nuclear gene, $R f-1$, that restores the BT-type cytoplasmic male sterility in rice (Oryza sativa L.) by PCR-based markers. Euphytica 129: 241247.

Konieczny, A. and F.M.Ausubel (1993) A procedure for mapping Arabidopsis mutations using co-dominant ecotype-specific PCRbased markers. Plant J. 4: 403-410.

Kurata, N., Y.Nagamura, K.Yamamoto, Y.Harushima, N.Sue et al. (1994) A 300 kilobase interval genetic map of rice including 883 expressed sequences. Nature Genet. 8: 365-372.

Lander,E.S., P.Green, J.Abrahamson, J.Barlow, M.J.Daly, S.E.Lincoln and L.Newburg (1987) MAPMAKER: an interactive computer package for constructing primary genetic linkage maps of experimental and natural populations. Genomics 1: 174-181.

Livak,K.J. (1999) Allelic discrimination using fluorogenic probes and the $5^{\prime}$ nuclease assay. Genet. Anal. 14: 143-149.

Michaels,S.D. and R.M.Amasino (1998) A robust method for detecting single-nucleotide changes as polymorphic markers by PCR. Plant J. 14: 381-385.

Murray,M.G. and W.F.Thompson (1980) Rapid isolation of high molecular weight plant DNA. Nucleic Acids Res. 8: 4321-4325.
Nasu,S., J.Suzuki, R.Ohta, K.Hasegawa, R.Yui, N.Kitazawa, L.Monna and Y.Minobe (2002) Search for and analysis of single nucleotide polymorphisms (SNPs) in rice (Oryza sativa, Oryza rufipogon) and establishment of SNP markers. DNA Res. 9: 163-171.

Neff,M.M., J.D.Neff, J.Chory and A.E.Pepper (1998) dCAPS, a simple technique for the genetic analysis of single nucleotide polymorphisms: experimental applications in Arabidopsis thaliana genetics. Plant J. 14: 387-392.

Sasaki,T. (2001) The progress in rice genomics. Euphytica 118: 103111.

Shen, Y.J., H.Jiang, J.P.Jin, Z.B.Zhang, B.Xi, Y.Y.He, G. Wang, C.Wang, L.Qian, X.Li, Q.B.Yu, H.J.Liu, D.H.Chen, J.H.Gao, H.Huang, T.L.Shi and Z.N.Yang (2004) Development of genome-wide DNA polymorphism database for map-based cloning of rice genes. Plant Physiol. 135: 1198-1205.

Tsunematsu,H., A.Yoshimura, Y.Harushima, Y.Nagamura, N.Kurata, M.Yano, T.Sasaki and N.Iwata (1996) RFLP framework map using recombinant inbred lines in rice. Breed. Sci. 46: 279284.

Yamanaka,S., I.Nakamura, K.N.Watanabe and Y.Sato (2004) Identification of SNPs in the waxy gene among glutinous rice cultivars and their evolutionary significance during the domestication process of rice. Theor. Appl. Genet. 108: 1200-1204.

Yano,M. (2001) Genetic and molecular dissection of naturally occurring variation. Curr. Opin. Plant Biol. 4: 130-135. 